Годишњак Филозофског факултета у Новом Саду, Књига XLVI-3 (2021)

Annual Review of the Faculty of Philosophy, Novi Sad, Volume XLVI-3 (2021)

Gorana Prodanović*

Faculté de Philosophie et Lettres

Université de Novi Sad

Institut français de Serbie, antenne de Novi Sad
UDK : 821.133.1-31.09 Mathieu N.

DOI: $10.19090 /$ gff.2021.3.355-367

pregledni rad

\title{
LA REPRODUCTION SOCIALE DANS LE ROMAN LEURS ENFANTS APRÈS EUX DE NICOLAS MATHIEU**
}

Dans le roman Leurs enfants après eux, Prix Goncourt 2018, Nicolas Mathieu aborde le problème de la transmission des schémas comportementaux des parents à leurs enfants. Par l'intermédiaire de ses personnages et à l'aide de son procédé littéraire, il pose un regard quasi sociologique sur ce phénomène. Il serait impensable d'aborder ce sujet sans évoquer Pierre Bourdieu et son analyse des mécanismes de reproduction sociale. Pour ce faire, nous allons adopter la terminologie propre à Bourdieu : habitus, champ, capital, illusion, etc. Cette lecture croisée permettra de voir dans quelle mesure, ce roman d'apprentissage relève de l'illustration de la théorie du célèbre sociologue dont l'auteur lui-même se réclame, ce qui rend cette démarche d'autant plus justifiable. Dans le roman, Mathieu dresse le portrait d'une région lisière de la France, en proie à la désindustrialisation dans les années 1990, avec pour protagonistes des représentants de différentes classes sociales.

Mots clés : Bourdieu, classes sociales, reproduction sociale, habitus, capital, sociologie, éducation, roman d'apprentissage.

\section{INTRODUCTION}

La pensée de Pierre Bourdieu a profondément marqué la société française et cela apparaît clairement dans le roman de Nicolas Mathieu - Leurs enfants après eux, prix Goncourt 2018. Avec ce roman, l'auteur transmet le message que chacun est enfermé à l'intérieur de sa classe sociale, incapable de

\footnotetext{
*gorana.prodanovic@gmail.com

${ }^{*}$ Cet article a été réalisé dans le cadre du projet scientifique Les langues, les littératures et les cultures romanes et slaves en contact et en divergence ( $\mathrm{n}^{\circ}$ 81/1-17-8-01) soutenu par l'AUF (Agence universitaire de la Francophonie) et l'Ambassade de France en Serbie et il contribue également au projet scientifique $\mathrm{n}^{0}$ 01600, financé par le ministère de l'Éducation, de la Science et du Développement technologique de la République de Serbie.
} 
s'en extirper, malgré toutes ses tentatives. Et pour employer un terme cher au célèbre sociologue, disons que le lecteur est confronté au problème de la reproduction sociale. Ceci signifie que les modèles familiaux se transmettent de génération en génération - en boucle. Même si la théorie bourdieusienne a été critiquée ou développée par la suite, cet ouvrage y reste éminemment fidèle. Le sociologue et le romancier ont en commun leur origine sociale modeste, la vue d'en bas, la réussite scolaire, mais aussi le poids qu'ils attribuent à la reproduction sociale, à l'instruction et à la sociologie.

\section{NICOLAS MATHIEU ET LEURS ENFANTS APRÈS EUX}

Après un premier roman noir, Aux animaux la guerre (2014), ce jeune auteur fait paraître Leurs enfants après eux (2018) qui est à la fois un roman d'apprentissage et un roman social. Il s'inscrit dans la lignée des romans d'apprentissage qui remonte au XVIII ${ }^{\text {e }}$ et surtout au XIX ${ }^{\mathrm{e}}$ siècle et aux œuvres de Balzac, Flaubert, Stendhal, Vallès sans oublier Gary, Duras, Tournier et de nos jours, Édouard Louis. S'il convient de constater que le roman Leurs enfants après eux appartient au genre du roman de formation, c'est du fait qu'il traite d'une période de jeunesse qui s'étend sur plusieurs années: le personnage principal est « jeune à crever » (Mathieu, 2018 : 27) et l'on suit sa trajectoire de 1992 à 1998. En outre, cet ouvrage s'apparente également au roman social pour son nombre important de personnages issus de la très fragile classe populaire. Quoiqu'il s'agisse d'un roman écrit à la troisième personne, on y retrouve des consonnances autobiographiques car la région et l'époque où se déroule l'action ainsi que la condition sociale d'Anthony Casati, personnage principal, coïncident avec celles de la jeunesse de l'auteur.

D'origine modeste, l'écrivain a ressenti la friction des classes sociales lors de sa scolarité : «À l'époque ce n'était évidemment pas le mot que je mettais là-dessus. Depuis j'ai lu Bourdieu et surtout Annie Ernaux.» (Abescat, 2018, para. 5). Mathieu lui-même se réclame donc ouvertement du sociologue ainsi que d'Annie Ernaux dont les romans regorgent de personnages issus de ce milieu social. À propos d'Ernaux on peut également évoquer l'inspiration bourdieusienne et son expérience de quelqu'un qui a vécu dans un «milieu dominé » et qui a fait "une expérience précoce et continue de la réalité des luttes de classes» (Ernaux, 2003: 69). Un autre indice de l'influence qu'elle a subie du sociologue serait sa contribution au volume Bourdieu et la littérature (auquel ont aussi contribué Pierre 
Bergounioux et d'autres écrivains) dont l'objectif était de dépister l'héritage du sociologue dans le champ littéraire. Dominique Viart, de son côté, met en avant le déterminisme social présent dans les romans d'Ernaux et sa manière de le dépasser, ainsi que sa condition de transfuge de classe. (Viart, 2014 : 28).

Ce ne sont que quelques traces de l'héritage de Pierre Bourdieu dont la théorie, d'ailleurs, a été remise en question et enrichie par les travaux de son disciple Luc Boltanski. Son "héritier» lui reproche de trop insister sur le déterminisme social et les rapports de domination, de croire que les agents sont trop aveuglés par leurs illusions (Faber, 2013 : 26-27). Malgré les développements et les critiques, la pensée bourdieusienne rayonne toujours, ce que met en évidence le roman Leurs enfants après eux. Nous allons essayer de le faire ressortir en utilisant la terminologie propre à Bourdieu : habitus, capital, espace social, illusio, etc.

\section{EN BOUCLE DANS LE ROMAN LEURS ENFANTS APRÈS EUX}

Le questionnement sous-jacent de l'œuvre de Pierre Bourdieu porte sur les rapports de domination, sur ce qui « fait que l'ordre du monde tel qu'il est [...] se perpétue en définitive aussi facilement, mis à part quelques accidents historiques, et que les conditions d'existence les plus intolérables puissent si souvent apparaître comme acceptables et même naturelles » (Bourdieu, 1998: 11). En essayant de remonter aux origines de cet ordre du monde, il se sert principalement des concepts d'habitus, de capital et de champ. Cela constitue le noyau de sa théorie élaborée au fil du temps.

Ces rapports de domination se retrouvent dans les schémas comportementaux qui se reproduisent en boucle et, se transmettant de génération en génération, tiennent les personnages enfermés chacun dans son propre milieu social, ce que Mathieu ne manque pas de faire remarquer :

Quoi qu'il en soit, les filles Mougel, leurs mecs, les cousins, les belles-familles, c'était le même monde. Il suffisait pour s'en rendre compte de voir le fonctionnement, dans les mariages, aux enterrements, à Noël. Les hommes parlaient peu et mouraient tôt. Les femmes se faisaient des couleurs et regardaient la vie avec un optimisme qui allait en s'atténuant. Une fois vieilles, elles conservaient le souvenir de leurs hommes crevés au boulot, au bistrot, silicosés, de fils tués sur la route, sans compter ceux qui s'étaient fait la malle. (Mathieu, $2018:$ 11) 
Le principe de la reproduction sociale que l'auteur pointe du doigt est identifiable à plusieurs niveaux : à travers le titre, l'exergue, l'agencement du récit et l'évolution des personnages.

Le titre même du roman, Leurs enfants après eux, condense au mieux son message. Il est emprunté au livre de Siracide (l'Ancien Testament) et fait partie des versets que l'on retrouve dans l'exergue du roman :

Il en est dont il n'y a plus de souvenir,

Ils ont péri comme ils n'avaient jamais existé ;

Ils sont devenus comme s'ils n'étaient jamais nés,

Et, de même, leurs enfants après eux.

Siracide, 44, 9 (Mathieu, 2018 : 6).

Outre le titre et l'exergue, les événements du récit sont agencés de manière à évoquer une certaine idée de circularité: les mêmes images apparaissent au début et à la fin du roman. Le roman s'ouvre sur la noyade d'un bachelier le 14 juillet 1991, et vers la fin du roman Patrick Casati, père d'Anthony, se noie le 14 juillet 1998. Et puis, au début du roman, Hacine Bouali vole la moto à Anthony, et à la fin, Anthony, à son tour vole la moto à Hacine. Enfin, la toute première image et la toute dernière, sont celles d'Anthony sur la plage appelée Déchetterie. La boucle se referme, il y a un éternel recommencement des faits et gestes.

Cette reproduction des hiérarchies sociales s'exerce aussi dans la perpétuation du récit familial, des rapports au sein de la famille, des choix personnels. Une multitude d'exemples prouvent que les personnages héritent de l'habitus intellectuel, moral, corporel de leurs parents à travers différentes injonctions et situations, et qu'il leur est difficile, voire impossible, de s'en débarasser. Même si pendant l'adolescence, les individus essaient de fuir leurs modèles familiaux, ils finissent par y succomber. Le style de vie des parents présage celui des enfants. C'est surtout visible chez Malek et Hacine Bouali, Patrick et Anthony Casati, Stéphanie Chaussoy et ses parents. Vanessa Léonard fait cependant exception, ce que nous allons montrer ultérieurement.

\subsubsection{Malek et Hacine Bouali}

$\mathrm{Au}$ plus bas de l'échelle sociale, la classe ouvrière est incarnée par la famille Bouali, issue de l'immigration marocaine. Le père Malek vit plus que modestement. Il appartient à la classe culturellement et économiquement 
dominée. Cette domination est extériorisée dans son corps prématurément ruiné : «Le garçon se tourna vers son père dont la pupille avait pris un contour hésitant, une couleur opaline qui signalent d'ordinaire la vieillesse. Il n'avait pourtant que cinquante-neuf ans » (Mathieu, 2018 : 61). Malek Bouali a travaillé dans la sidérurgie. Lui-même obéissant, mais ne pouvant se faire obéir qu'à coups de bâton, est incapable même de transmettre son habitus moral. Son fils a honte de lui : "À chaque fois qu'il ouvrait la bouche, Hacine avait envie de se planquer» (Mathieu, 2018: 62). La désindustrialisation ayant fait son effet, Hacine désœuvré nourrissait l'ambition de se défaire de cette position de dominé en s'enrichissant comme dealer de drogue :

Hacine mesurait les avantages de sa situation par rapport à celle de ses parents. En dehors même de tout le fric qu'il se faisait, lui n'avait pas à se farcir la durée, la routine, cette répétition dissolvante, du lundi au vendredi, en attendant les vacances, selon un cycle inlassable qui vous menait de la jeunesse au cimetière en un claquement de doigts (Mathieu, 2018 : 212).

Au cours de sa carrière de dealer il n'a pas hésité à avoir recours à la violence, mais les mauvaises fréquentations le rattrapent, et il finira comme petit vendeur dans un magasin, pitoyable et dominé :

Les peines se compensaient par des achats d'électroménager, la longueur des jours venait butter sur la perspective des vacances, Coralie effaçait la monotonie de la semaine et les potes, la beuh, l'abonnement Canal et Tomb Raider faisaient le reste. L'ensemble composait au bout du compte une petite vie bien acceptable (Mathieu, $2018: 299$ ).

Ce qu'il y a de plus représentatif de cette classe sociale, ce sont : la cité, la drogue, la détérioration précoce du corps, les rapports familiaux détériorés, les steaks hachés, les patates, la soumission, la violence, le mutisme, la misère.

\subsubsection{Patrick et Anthony Casati}

Anthony, le personnage principal, fils unique de Patrick Casati, ouvrier, et Hélène Mougel, employée de bureau, se trouve ainsi à cheval entre la classe moyenne et la classe ouvrière. Chez eux, on habite des maisons pavillonnaires dont le sol est recouvert de moquette ou de lino, avec des posters sur les murs, et modestement meublés. Patrick Casati ne possède qu'une part infime d'un 
capital insignifiant. Lui-même, enfant de parents peu instruits, il est fragilisé par la fermeture de l'usine, colérique, il vit sous l'emprise de l'alcool, et sa vie est une lente descente aux enfers, un déclassement social total. Anthony Casati est amoureux de Steph, petite bourgeoise qui est hors de portée pour lui. Il rêve de quitter sa ville natale, ce qu'il fait effectivement, mais il y revient, au bout de deux ans, réformé par l'armée. Par la suite, il ne fait que marcher sur les pas de son père : sillonner la vallée à moto, gagner médiocrement sa vie et ingurgiter d'importantes quantités de bière. Au travail, ses collègues lui ressemblent et partagent la même honte de leur soumission :

Tous partageaient le même genre de loisirs, un même niveau de salaire, une incertitude identique quant à leur avenir et cette pudeur surtout, qui leur interdisait d'évoquer les vrais problèmes, cette vie qui se tricotait presque malgré eux, jour après jour, dans ce trou perdu qu'ils avaient tous voulu quitter, une existence semblable à celle de leurs pères, une malédiction lente. Il ne pouvait admettre cette maladie congénitale du quotidien répliqué (Mathieu, $2018: 371)$.

Cet univers est envahi par la violence, la dépression, le mutisme, la télévision, les motos, l'excès d'alcool, la bière...

\subsubsection{La famille Chaussoy: Stéphanie et ses parents}

Les Chaussoy sont une famille de la petite bourgeoisie grâce à laquelle Stéphanie bénéficie d'un capital corporel, économique et symbolique, mais démunie de capital culturel. Son père, concessionnaire Mercedes, avait abandonné ses études de médecine. Sa mère avait abandonné ses études de droit. Stéphanie est fainéante au lycée jusqu'au jour où son père, obsédé par le besoin d'accumuler des capitaux, va s'acharner sur elle. Sous sa pression, au prix d'un énorme investissement personnel, elle parvient à s'inscrire à l'ESSEC, après s'être approprié l'illusio nécessaire :

Enfin, il y avait les cadors, qui se prévalaient d'une bonne mention et d'un dossier béton, véritable rampe de lancement pour les carrières désirables. Ceux-là emprunteraient des canaux étroits et, mis sous pression, iraient vite, grimperaient très haut. [...] Steph voulait faire partie de cette [...] catégorie (Mathieu, 218: 313).

Malgré de très bons résultats, Steph ne persiste pas dans ses études. Elle décide de suivre son amoureux au Canada. C'est l'amour qui l'emporte sur 
l'instruction. Elle reproduit ce qu'elle a connu : le modèle du couple et le manque de motivation pour les études. Ainsi Stéphanie Chaussoy finit par succomber à son habitus familial.

L'aisance, le confort, la politique, l'investissement dans la progéniture, les études, les bijoux, la pelouse, la piscine, le whisky et autre peuplent cet univers de la petite bourgeoisie.

\subsection{Vanessa Léonard - transfuge de classe}

Vanessa Léonard fait partie de la classe moyenne mais elle se croit supérieure. Ce qui caractérise la classe moyenne, ce sont les maisons pavillonnaires, la vie familiale, les vacances toujours au même endroit, le travail dans l'administration, les balades, les jeux de grattage, les chemisettescravates, la satisfaction dans la médiocrité : « [...] favorables à l'état des choses, modérément scandalisés, inquiets des périls télévisés, satisfaits des bons moments que leur offre la vie... » (Mathieu, 2018 : 189).

C'est souvent devant le poste de télévision que Mathieu place ses personnages issus des classes populaire et moyenne. Dans son ouvrage Sur la télévision, Bourdieu a dénoncé le rôle des médias. Il y constate que la télévision a eu pour effet d'enfermer les classes culturellement défavorisées dans leur propre culture sans leur permettre l'accès à la « culture légitime » :

La télévision a une sorte de monopole de fait sur la formation des cerveaux d'une partie très importante de la population. Or, en mettant l'accent sur les faits divers, en remplissant ce temps rare avec du vide, du rien ou du presque rien, on écarte les informations pertinentes que devrait posséder le citoyen pour exercer ses droits démocratiques (Bourdieu, $1996: 18$ ).

À un moment donné, Vanessa comprend que le niveau d'instruction fait souvent pencher la balance sociale. Elle se met au travail, réussit le bac avec mention, part faire des études de droit à Paris et parvient à s'y faire une place. Dans le roman, Vanessa est le seul contre-exemple de la reproduction des classes sociales. Elle est un transfuge de classe.

À travers son exemple, l'auteur indique une piste qui permettrait de s'arracher à son milieu social - l'éducation scolaire. Dans ce roman, ceux qui parviennent à réaliser leur objectif, fuir leur ville natale, sont ceux qui ont misé sur la réussite scolaire. C'est le cas de Steph et de Vanessa. Malheureusement, cela ne réussit pas toujours. La noyade du bachelier au début du roman atteste 
du contraire. D'origine modeste, il obtient une mention "très bien » au baccalauréat mais finit englouti par le lac. Le lac symbolise la société qui de toute façon l'aurait entravé en raison de ses origines.

\subsection{Espace social et effets unificateurs}

Dans le roman on constate que l'espace social est fortement connoté et que les membres de différentes classes ne se croisent que rarement. Ce compartimentage de l'espace est le mieux illustré dans l'exemple du lac : la plage la Déchetterie à proximité d'une sortie d'égout ; la plage américaine, pour les grosses têtes; le club nautique, le plus bel endroit, etc. (Mathieu, 2018 : 15). Si comme par magie il arrive aux jeunes issus de différents milieux de succomber temporairement à leurs tentations, les rencontres se passent dans des zones anonymes et inhabitées : centrale électrique désaffectée, au pied de la statue qui surplombe la vallée ou sur la route au milieu de nulle part. Dans les zones où la société est absente, tout est possible.

Les moments forts peuvent parfois assumer un rôle unificateur. Dans le roman, les gens ne se sentent en parfaite communion qu'à l'occasion du 14 juillet, à l'enterrement d'un ancien syndicaliste, à la demi-finale de la Coupe du monde en 1998. En fait, c'est justement grâce à ces moments-là que l'on prend conscience de la ségrégation inhérente à l'espace social. Et puis, le choix de l'auteur de ne représenter les événements que pendant l'été, période de l'année où on circule plus librement, participe à son envie d'abattre ces barrières sociales afin de rendre possible le contact entre les personnages de différentes origines.

\section{L'ÉDUCATION SCOLAIRE}

Outre la reproduction sociale, l'importance attribuée à l'instruction est donc un autre point commun entre Mathieu et Bourdieu. Depuis Les Héritiers (ouvrage rédigé avec Jean-Claude Passeron, publié en 1964) Bourdieu a fait beaucoup de recherches sur l'égalité des chances dans le cadre scolaire :

La méconnaissance des déterminants sociaux de la carrière scolaire - et, par là, de la trajectoire sociale qu'elle contribue à déterminer - confère au titre scolaire la valeur d'un droit de nature, et fait de l'école une des instances fondamentales du maintien de l'ordre social (Bourdieu, 1979 : 452). 
Il observe que les familles privilégiées investissent dans l'éducation de leurs enfants, ce qui «permet de comprendre aussi que les plus hautes positions sociales, soient de plus en plus complètement monopolisées par les enfants des catégories privilégiées [...]» (Bourdieu, 1994: 40). À titre d'exemple, dans le roman en question, juste avant le baccalauréat, Steph prend conscience de l'importance d'un projet de réussite scolaire :

Elle avait saisi le fonctionnement général, sur le tard, mais quand même. L'école faisait office de gare de triage. Certains en sortaient tôt, qu'on destinait à des tâches manuelles, sous-payées, ou peu gratifiantes. Il arrivait certes que l'un d'entre eux finisse plombier millionnaire ou garagiste plein aux as, mais dans l'ensemble, ces sorties de route anticipées ne menaient pas très loin (Mathieu, $2018: 312$ ).

Stéphanie découvre les parcours scolaires qui engendrent les élites. Illuminée, elle admire les étudiants de l'ESSEC (École supérieure des sciences économiques et commerciales), une des plus prestigieuses grandes écoles :

Ceux qui comprenaient intimement la machine et parlaient la langue de leur temps, ceux qui embrassaient exactement une époque perpétuellement accélérée, exponentielle par nature, dévorante, infusée de lumière, de vitesse et d'argent, ils étaient là, princes économistes, meneurs d'affaires, avec leurs chemises bleues, leur corps lisse, flûté, leur épouvantable allant (Mathieu, $2018: 319)$.

Selon Bourdieu, les grandes écoles donnent accès au champ du pouvoir, au champ des champs, capable de légitimer quoi que ce soit. Inversement, plus bas l'individu se trouve quelque part sur l'échelle sociale, et il a moins de possibilité de création en raison de son manque de capital culturel. Hacine Bouali, ne possédant aucun capital, en est un exemple éclatant. Rien que la vue sur la ville depuis son nouvel appartement inspire à Hacine des idées sur la marche du monde :

La vue était splendide, on voyait toute la ville et loin vers Guérémange, à l'est. Curieusement, ce panorama lui filait le bourd on. À regarder les autres de haut, telles des fourmis, on en venait à se poser des questions d'ordre général (Mathieu, $2018: 300$ ). 
En analysant l'œuvre de Pierre Bourdieu, Champagne et Christin ont constaté : «Changer le regard sur le monde [...], c'est changer la réalité sociale » (Champagne, Christin, $2012: 144$ ).

\section{SOCIOLOGIE}

Dans le roman, un petit clin d'œil rend hommage à la sociologie à travers le personnage de Vanessa : «Peut-être que l'illumination était venue en cours de socio, ou en faisant les courses au Leclerc avec sa mère » (Mathieu, 2018 : 190). La sociologie a donc pour rôle d'éclairer l'individu sur les lois de la société. Durkheim déjà, puis Bourdieu à son tour, étaient convaincus de l'utilité de cette science : elle devrait être "l'instrument de libération » surtout des classes culturellement défavorisées. Les deux sociologues reconnaissent pourtant comme un problème la diffusion de ce savoir et son jargon spécifique incompréhensible aux profanes. Un autre écueil de la diffusion serait une vulgarisation trop simpliste qui tuerait le message. Toutefois, le message du sociologue est passé par le biais du roman Leurs enfants après eux qui démonte ces fins mécanismes sociaux et qui s'adresse aux profanes dans un langage quotidien tout en évitant cet écueil de la banalisation.

\section{CONCLUSION}

Le sociologue Pierre Bourdieu, par son œuvre foisonnante, et Nicolas Mathieu, par son roman Leurs enfants après eux, démontrent qu'un rouage social complexe tient l'individu enfermé au sein de son milieu social. Mathieu insiste sur cette reproduction des hiérarchies sociales à travers son procédé littéraire en représentant certains événements en boucle et les personnages du père Malek Bouali et du fils Hacine Bouali, du père Patrick Casati et du fils Anthony Casati, des parents Chaussoy et de leur fille Stéphanie. Bourdieu l'explique par des concepts d'habitus, de capital, de champ. S'il est vrai que certaines personnes des milieux populaires réussissent leur vie, c'est grâce à l'accumulation du capital culturel ou bien elles dérogent à la règle. Un cas exceptionnel est représenté par Vanessa Léonard qui, ayant pris conscience de l'importance des études, quitte sa ville natale et donne une preuve que l'ascension sociale est quand même possible. En Vanessa, on reconnaît Pierre Bourdieu et Nicolas Mathieu qui ont, l'un comme l'autre, accédé à la réussite sociale malgré leur origine modeste grâce à l'éducation scolaire et à une prise 
de conscience sociale, et qui, de cette façon, sont eux-mêmes les meilleurs exemples de ce qu'est le transfuge de classe.

Gorana Prodanović

ON A LOOP IN THE NOVEL AND THEIR CHILDREN AFTER THEM BY NICOLAS MATHIEU

Summary

In the novel And Their Children After Them, Prix Goncourt 2018, Nicolas Mathieu points out the transmission of behavioral patterns from parents to their children. With his characters and his literary process, he views this phenomenon from an almost sociological standpoint. It would be unthinkable to approach this subject without mentioning Pierre Bourdieu and his analysis of the mechanisms of social reproduction. In doing so, we will therefore use Bourdieu's terminology: habitus, capital, field, illusio, etc. This cross-reading will allow us to see to what extent this bildungsroman illustrates the theory of the famous sociologist. The fact that the author himself refers to Bourdieu further justifies our approach. In the novel, Mathieu draws a portrait of France on the outskirts in the throes of deindustrialisation in the 1990s, with representatives of different social classes as protagonists.

Key words : Bourdieu, social classes, social reproduction, habitus, capital, sociology, education, bildungsroman.

\section{RÉFÉRENCES BIBLIOGRAPHIQUES}

Abescat, M. (2018, 7 novembre). Prix Goncourt 2018 : comment Nicolas Mathieu a écrit son roman "Leurs enfants après eux". Consulté le 5 février 2021, disponible sur https://www.telerama.fr/livre/le-baumelitteraire-de-nicolas-mathieu,n5837621.php

Baudorre, Ph.-Rabaté, D. \& Viart, D. (dir.) (2007). Littérature et sociologie. Pessac : Presses Universitaires de Bordeaux.

Best, F.-Blanckeman, B. \& Dugast-Portes, F. (dir.) (2012). Annie Ernaux, le Temps et la Mémoire. Paris : Stock.

Boltanski, L. (2009). De la critique. Précis de sociologie de l'émancipation. Paris : Gallimard.

Bourdieu, P. (1979). La distinction: critique sociale du jugement. Paris : Éditions de Minuit.

Bourdieu, P. (1980). Le sens pratique. Paris : Editions de Minuit.

Bourdieu, P. (1989). La noblesse d'État : grandes écoles et esprit de corps. Paris : Éditions de Minuit. 
Bourdieu, P. (1992a). Što znači govoriti : ekonomija jezičnih razmjena. Zagreb : Naprijed.

Bourdieu, P. (1992b). Les règles de l'art : genèse et structure du champ littéraire. Paris : Seuil.

Bourdieu, P. (1994). Raisons pratiques : sur la théorie de l'action. Paris : Seuil.

Bourdieu P. (1996). Sur la télévision suivi de L'emprise du journalisme. Paris : Liber.

Bourdieu, P. (1998). La Domination masculine. Paris : Seuil.

Burdije, P. (2000). Narcisovo ogledalo : rasprava o televizijskom novinarstvu. Beograd : Clio.

Burdije, P.-Paseron, Ž.-K. (2014). Reprodukcija : elementi za jednu teoriju obrazovnog sistema. Beograd : Fabrika knjiga.

Burdije, P. (2019). Nacrt za autoanalizu. Loznica : Karpos.

Champagne, P.-Christin, 0. (2012). Pierre Bourdieu : une initiation. Lyon : Presses universitaires de Lyon.

Chardin, Ph. (dir.) (2007). Roman de formation, roman d'éducation dans la littérature française et dans les littératures étrangères. Paris : Kimé.

Ernaux, A. (2003). L'écriture comme un couteau. Entretien avec Frédéric-Yves Jeannet. Paris : Stock.

Faber, A. (2013). Les fondements de la critique sociale chez Pierre Bourdieu et Luc Boltanski. In: Kane, O.-George, É. (dir.) (2013). Où (en) est la critique en communication. Le Centre de recherche GRICIS. Disponible sur https://www.cricis.uqam.ca/wp-content/uploads/IMG/pdf/ ActesColloqueOu-_en_-est-la-critique-encommunication_Gricis2012_Reduit.pdf

Fourny, J. (1994). Les littératures de Pierre Bourdieu. L'Esprit Créateur, vol. 34, n 1, 92-100. Disponible sur http://www.jstor.org/stable/26287611

Martin, J-P (dir.). (2010). Bourdieu et la littérature. Suivi d'un entretien avec Pierre Bourdieu. Nantes : Cécile Defaut.

Mathieu, N. (2018). Leurs enfants après eux. Arles : Actes Sud.

Nemanjić, M.-Spasić, I. (ed.) (2006). Nasleđe Pjera Burdijea : pouke i nadahnuća. Beograd : Institut za filozofiju i društvenu teoriju : Zavod za proučavanje kulturnog razvitka.

Petrov, A. (2015). Pjer Burdije. Beograd : Orion art : Fakultet za medije i komunikacije. 
Saint-Amand, D. (2010). Ce que Bourdieu fait à la littérature. Acta fabula, vol. $11, \mathrm{n}^{\circ}$ 9, Essais critiques, Octobre 2010. Disponible sur http://www.fabula.org/revue/document5963.php

Škorić, J.-Kojić, M. (2016). Konfliktni pristup obrazovanju sagledan kroz teoriju Pjera Burdijea. In : Pedagoška stvarnost: časopis za školska i kulturnoprosvetna pitanja, 1, 47-60.

Viart, D.-Vercier, B. (2008). La littérature française au présent : héritage, modernité, mutations. Paris : Bordas. 